\title{
LA IMPORTANCIA DE LOS PERSONAJES EN EL DISEÑO NARRATIVO: \\ LA FIGURA DE LANARK/THAW COMO ELEMENTO DE ANALISIS EN LA OBRA DE ALASDAIR GRAY LANARK
}

\author{
Mario DIAZ MARTINEZ \\ Universidad de Lein
}

\section{RESUMEN:}

The contemporary literary critics have seriously questioned the role played by the characters in the narrative discourse of today. However, if we adopt a literary approach opened to the idea of intertextuality as a general principle (which is also based on the postmodernist textual theories), it is possible to apply the same phenomena to the network ol' relationships established between the very characters of a work, as a starting point to analize the work in which they exist. After stating brielly these theoretical conceps from the point of view of well-known scholars, we move on towards the figure of the main character in Alasdair Gray's Lanark, analizing its importance in the wide context of the liction discourse. So, Lanark/Thaw, as a single entity composed by two «realities», reveals itself as a ligure with a strong intra-textual component, that is, with a double existence in two different levels of conscioussness. We also detail its relationsips with other minor characters which populate the worlds of Lanark, and finally we are capable to explain its influence in the work as a whole.

\section{PALABRAS Cl.AVE:}

Personaje - Intertextualidad - Alasdair Gray - Ficción - Lanark - Doble Identidad - Interfiguraloidad

\section{1.-EL ESTUDIO DEL PERSONAJE:}

El siglo XX ha resultado una época especialmente violenta y dramática, que nos ha brindado la oportunidad de asistir a la declaración de muchas muertes: Dios, el humanismo, la tragedia y también, cómo no, la muerte del personaje. Muchos novelistas modernos han negado de raiz las características tradicionales del personaje, modeladas según una visión tradicional del hombre. Alain Robbe-Grillet, por ejemplo, rechazó categóricamente la concepción de «El mito arcaico de la profundidad» y con él la potencialidad psicológica de los personajes ${ }^{\prime}$, mientras que Helene Cixous cuestiona no sólo la estabilidad sino también la unidad del personaje. Según ella el «Yo»

Robbe-Gillet, A; Pour un Nouveau Roman, Paris: Gallimard, 1963, 
es siempre más que uno, diverso, capaz de ser todos aquellos que alguna vez serä, como un grupo actuando a la ve $z^{2}$. lo que se maniliesta especialmente en Lanark.

Finalmente los postulados estructuralistas vinieron a rumatar la cuestión, ya que la concepción del personaje se ajustaba difícilmente a sus teorías, elaboradas a partir de una ideología que rechazaba al hombre como centro de estudio y se enfrentaba abiertamente a las nociones de individualidad y hondura psicológica:

«Stress on the interpersonal and conventional systems which traverse the individual, which make him a space in which forces and events meet rather than an individuated essence, leads to a rejection of a prevalent conception of character in the novel: that the most succeslul and «living» characters are richly delineated autonomous wholes, clearly distinguished from others by physical and psychological peculiar characteristics. The notion of character, as the structuralists would say, is a myth. $p^{3}$

Io que sin embargo nadie puede negar es el hecho de que las relaciones existentes entre personajos de textos diversos constituyen una de las dimensiones más importantes del fenómeno de la intertextualidad (entendido bien desde una perspectiva post-estructuralista como trampolín que conduce hacia especulaciones asociativas sobre cultura y semiótica, bien desde el punto de vista del tradicional estudio literario como las estructuras $e$ interrelaciones de textos literarios entre si) ${ }^{*}$

\section{2.-EL COMPONENTE INTERTEXTUAL:}

Podemos considerar un texto como una estructura autónoma de signos, delimitada por un inicio y un linal y coherente por la deliberada interrelación de sus constituyentes. Un intertexto estaría cartcterizado por atributos que exceden la definición anterior, en el sentido de que por una parte sus constituyentes están referidos a otros textos y por otra su colnerencia presenta tanto un aspecto intratextual que garantiza la integridad del texto como otro intertextual que genera relaciones estructurales con otros textos. En base a esta argumentación se podría plantear una escala de mayor 0 menor intertextualidad: siendo la idea dominante la autonomia textual se generaría un texto atutocontenido frente al planteamiento de maxima

2 Cixous, Helene; “The Charncter of aChnracterm, New Luerary Studies, New York, 1974, pp.383-402

Barthe. Roland; Structuralist Poctics. Structuralism. Linguistics and the Study of Literature. London, Routledge \& Kegan Paul, 1975, p.230

PJett, Heinrich (ed.): Interfextuality, Berlín: Walter de Gruyter. 1991, pp. 
intertextualidad cuyo postulado sería que «Todo texto es intertexto»5. Barthes reconoce que una de las vías que permiten la deconstrucción del lenguaje radica en la permutación de textos que existen o han existido:

"Any text is an intertext; other texts are present in it, at varying levels, in more or less recognisable forms: The texts of the previous and surrounding culture., ${ }^{6}$

Claros ejemplos de intertextualidad serían obras tan celebradas como Lost in the Fun House de John Barth, el Dr. Faustus de T. Mann o Rosencratz and Guildenstern Are Dead de Stoppard. Resulta sorprendente, a la vista de la trascendencia de este tema y su frecuencia en la literatura de nuestros dias, la escasa atención que se le ha dedicado hasta el momento en la crítica contemporánea y en la propia teoría de la intertextualidad. Quizá una de las razones para que exista tal laguna sea la desconlianza general que muchos estudiosos sienten hacia el trabajo centrado en los personajes, sobre todo aceptando los planteamientos del New Criticism, con su énfasis en la concepción del texto como unidad autosuficiente y autocontenida, marco estanco en el cual se dan partes funcionaimente relacionadas, o de teúricos como Culier, para quien los personajes no son sino un aPrejuicio Ideológico» más que un tema de investigación respetable. ${ }^{\text {? }}$

La intertextualidad se puede extrapolar también a los personajes que Alasdair Gray recrea en su obra Lanark, puesto que no estan tlegidos al azar sino que se halian firmemente anclados en un contexto cultural y literario de carácter eminentemente Escocés (Monboddo, Lanark, Duncan, Noakos y muchos otros) al que se hace referencia en ocasiones paródicamente para aportar al lector nuevas pistas y perspectivas de la obra en conjunto. Los personajes de Gray no son sino construcciones literarias, creaciones lingǘsticas dignas de estudio e inextricablemente unidas al discurso en el que habitan. Como postula Barthes:

"The character and the discourse are each other's accomplices and it is as wrong to suppress the character as it is to take him off the page in order to turn him into a psychological character (endowed with possible motives)m."

[eitch, Vineont; Deconstructive Criticism: An Aduanced Introduction. London: Ilutchituson, 1983.

"Barthes, Roland; "Theory of the 'Text" in Robort Young (ed.) Untying the Text: A Post-Structuraltst Reading; London: lloutledge \& Kegan Paul, 1981, p.39.

${ }^{7}$ Culler, Jonathan; Structuralist Poetics. London: Routledgo \& Kegan Paul, 1975.

${ }^{K}$ Barthes, Roland; $S / Z$, New York: Hill \& Wang, 1974, p.178 


\section{3.-NUEVOS PLANTEAMIENTOS EN EL ESTUDIO DEL PERSONA.JE:}

En los últimos años el silencio que envolvía al análisis de los personajes se estí rompiendo, comenzando a aparecer artículos y estudios como los de James Phelan, Mary Doyle Springer, Martin Price o Danicl Schwartz. Phelan, por ejemplo, clarifica los planteamientos distinguiendo entre «Funciones Miméticas» de un personaje (Que vendrían a ser los semas y rasgos propios que tienen importancia en términos narrativos) y las aDimensiones Miméticas» que se constituirían mediante aspectos superlluos a la narración pero ayudarían al establecimiento de la identidad individual del personaje."

Los trabajos de todos estos autores comparten desde diversas ópticas el reconocimiento de una perspectiva dual de los personajes como construcciones lingüisticas y como seres humanos imaginados. Para Barthes, esta tensión es dialécticamente positiva, ya que la narración y el personaje forman una matriz dinámica, sobre la que descansa el discurso:

«The discourse creates characters...not to make them play among themselves before us, but to play with them, to obtain a complicity which assures the uninterrupted play of the codes.. 11

De acuerdo a esta concepción los personajes son creados cuando "Identical semes traverse the same proper name several times and appear to settle on it»". Por tanto, son producto de la combinación de varias figuras complejas y/0 congruentes dispersas por el texto, cuya relación con el "personaje» que cvocan es una equivalencia entre "sign and sum», es decir, los personajes serían un conjunto de tórminos intersecantes que existen en un medio semántico y sintáctico más que en el tiempo y el espacio.

Aceptar estos postulados supone reconocer explícitamente que los personajes no tienen «motivos», y que es erróneo atribuirles ningún lipo de motivación, pero indudablemente el discurso en que se laallan inmersos y que les da sentido sí que estai motivado, puesto que debe establecer y justificar nuestra atención mediante un proceso de planteamiento de situaciones y resolución de enigmas. Las secuencias de signos que nos ocupan crean en nuestras mentes vividas criaturas de las cuales recibimos inputs de información y a las cuales damos una respuesta emocional como si fueran algo más que las liguras asépticas de un discurso. Aún cuando fuera posible, no resultaría fácil imaginar un Quijote sin D. Quijote y Sancho, ni a Lanark privada de los personajes que la habitan y dan forma. Cabría, por tanto, plantearse si las nuevas tcorias y planteamientos de nuestro siglo únicamente han cambiado los conceptos clásicos vigentes,

"Scheiber, Andrew; «Sign, Seme and The Psychological Character" The Journal of Narrative Technique, Vol.21, N'3, Fall 1991, pp.263-271

16 Barthes. Toland: S/Z, New York: Iliil \& Wang. 1974, pp. 178-9 
puesto que ante un corpus narrativo no podemos simplemente ignorar los personajes, a riesgo de autolimitar nuestra visión y privarnos de los múltiples matices y posibilidades quo se nos olrecen. Una lectura completa yenriquecedora de la narrativa de Gray exige una reflexión profunda sobre cada figura que aparece, su razón de ser, las perspectivas que aporta a la narración y su funcionamiento en el discurso global de la obri, de ahí el enfoque propuesto. Los personajes sugieren la existencia de dos polos contrapuestos: it la vez nos parecen «reales» e imaginarios, porque los efoctos de tales construcciones imaginativas dependen de su dependencia referencial, perosu signilïcación trasciende ese contexto. la riqueza de esta paradoja sugiere que el estudio y análisis de los personajes, definidos por Weinsheimer como ethe textualized persons, personified texts that are characters"' 12 no os una pérdida de tiempo, sino una puerta de estudio para la crítica contemporánea. Cuando el conjunto sémico que constituye la "realidad» de un personaje a la vez refuerza y cuestiona la autoridad del discurso que le contiene, produce lo que no deja de ser una típica paradoja como las que se hallan en la raiz de las teorías deconstruccionistas. Ante la tradicional dialéctica narración/personaje una vía de solución sería no aceptar ni que la narración se subordina al personaje ni viceversa, sino que ambos, narración y personaje, son interdependientes, tal como lo entiende Henry James: "What is character but the determination of incident? What Is Incident but the illustration of character?" 1.4

Los personajes, por tanto, se subordinarían a la narración cuando ésta fuera el centro de atención, pero del mismo modo la narracjón podría subordinarse al personaje on el momento que así lo decida el interés del loctor o la propia narración. Esta reversibilidad de jerarquías debiera caracterizar no sólo la lectura sino también la crítica y la teoría literaria.

\section{4.-LOS PERSONA.JES DE LANARK:}

\section{1.-La Identidad Lanark/Thaw:}

El papel desempeñado por Lanark como protagonista de la narraciön es de especial importancia para abordar la signifjeación de la obra en su conjunto. Cuando aparece por primera vez on la apocalíptica ciudad de Unthank no tiene apenas identidad propia, $y$ sin embargo se alegra de encontrarse totalmente solo, lo que resultará a la larga irónico, ya que posteriormente empleará todo su tiempo en intentar romper su aislamiento y soledad sin ningún éxito. Esta situacion aporta nuevos elementos al uso

"Bartios, Roland; $S / Z, p .95$

${ }^{12}$ Shlomith, Rimmon: Narrative Fiction: Contemporary Poetics; New York: Mathuen, 1988, p.34

${ }^{13}$ Morris (ed. ), Henry James: Selected Litorary Criticism. Harmondsworth:

Penguin. 1963, p.80 
paradójico que hace Gray de la técnica de arrojarnos directamente al centro de la acción, ya que en este caso nos encontramos en el centro de la «inacción» (por otra parte, lo que caracteriza al mundo de Unthank) con un personaje que es lo más parecido que podemos encontrar en términos humanos a la famosa "Tabula Rasa" de los filósofos empíricos, de tal manera que el personaje ha de tomar forma partiendo de cero únicamente en base a sus propias experiencias. Y sin embargo en este personaje aparentemente "vacio" vamos progresivamente a ir encontrando indicios que nos llevan a pensar que de una forma u otra debe haber sido anteriormente el joven Duncan Thaw, lo que explicaría el resto de la narración. Es posible reconocer ciertas trazas del carácter de Thaw que no han sido del todo borradas, como su desafiante cabezonería, o su anhelo insatisfecho de ternura y comprensión. Quizás estos rasgos tan determinantes hayan podido trascender su suicidio, su muerte ahogado, o su voluntaria inmersión en otra esfera de consciencia, posibilidades que quedan abiertas en el libro. Algunos otros puntos en común incluyen la necesidad de lograr una relación sexual satisfactoria con una mujer (que pronto desplazará la felicidad inicial de estar solo) y una permanente falta de seguridad personal a la hora de perseguir sus propósitos.

Sin embargo, la mayor evidencia de que Lanark y Thaw son en «realidad» una misma identidad aparece en el prólogo al final del tercer libro donde el invisible oráculo, que ha sido llamado precisamente para suplir a Lanark de la información que le falta sobre su pasado, tras presentarse a sí mismo, narra la vida de Duncan Thaw, explicando que se trata de la historia de Lanark en una vida anterior. Nos encontramos por tanto ante una fieción que evoca el tema de las dobles identidades o, para ser mas fieles al término original en inglés, la problemática del «alma dividida» («The divided soul»), algo por otra parte bastante tradicional en el contexto literario Escocés desde el Dr. Jekyll y Mr. Hyde de Stevenson. Esta referencia tomática parece confirmar las teorias de críticos como Wolfgang Müller, quienes afirman que existe una estrecha y compleja red de relaciones entre un universo de personajes de ficción de diferentes épocas y autores, sobre los cuales el autor-creador realizaría una labor de selección $y$ síntesis de peculiaridades y rasgos de unos y otros hasta contruir el arquetipo que mejor cuadro en la ficción, justificando en este proceso el concepto de sinterfiguraleidad».

El toque distintivo y peculiar de Gray en este ambito de la interfiguraleidad es el hecho de que se realiza no sólo intertextualmente sino también. aunque aparentemente resulte contradictorio, intratextualmente. Tal fenómeno, del cual existen más precedentes de los que en principio podríamos pensar, se produce cuando una obra literaria combina dos o más contextos de licción (mundos, on definitiva) y establece relaciones y paralelismos entre figuras aparentemente independientes de ambos, como en el caso de Thaw y Lanark. El ejemplo más claro de «interfiguraleidad" se produce en las obras literarias en las que aparece la uplay-within-a-play» como clave 
de la narración, a partir de la cual se crean intrincadas y a menudo reveladoras interrelaciones entre personajes de distintos planos de ficción, conducentes a un climax que posibilita la verdad última (Resulta inevitable pensar en Hamlel como cjemplo palpable). En Lanark, tal lunción se te asigna claramente al Oraiculo, si bien nuevamente nos vamos a encontrar la particularidad de que, si normalmente la aplay-within-a-play» convierte en un rellejo de algún modo distorsionado como "licción» de to que acontece en la arealidad» de la obra, en este caso sucede todo lo contrario: El mayor realismo on fondo y forma de la historia de Thaw es revelado en un contexto fantústico y grotesco como el Instituto.

\section{2.-El Fracaso de las Relaciones Humanas:}

En ciertos aspectos, las experiencias que vive Lanark son más enriquecedoras que las de Thaw, y prueba de ello es la capacidad de comprensión linal que adquiere Lanark, nunca soñada por Thaw. A pesar de ello, Lanark no es simplemente una descripción del progreso personal en la consecución de experiencias y sabiduría, ya que si bien el mundo de Lanark está más allả (en muchos sentidos) del de Thaw, el primero no puede comprender totalmente la personalidad y circunstancias del segundo, lo que conlleva que le sea imposible verse a sí mismo en esa personilicación anterior. Lanark se muestra especialmente desconecrtado (y Gray también) acerca de lo que realmente sucedió cuando Thaw parece asesinar al personaje de Marjory y atin más por la cuestión referente a si Thaw se suicidó a causa de una mera fantusía o debido a los remordimientos de un crimen real. Más signilicativo resulta el hecho de que, para Lanark, Marjory sea una persona totalmente diferente a la otra figura femenina de fima, mientras que para Rlma se trate de ella misma cuando tenia diecinueve años, lo que implica que el personaje de Rima es ol único capaz de reconocer su existencia como una continuación de un estado anterior.

Si para Marjory el personaje de Thaw es inseguro de sus posibilidades, no muy atractivo e introvertido, Rima mantiene hasta el linal que lanark (quien, a decir verdad, es bastante indulgente con sus propias acciones y motivos) es esencialmente egoista y egocéntrico, lo que no le hace muy digno do confianza. Varias veces le acusa de haberla apartado de él, a pesar de que Lanark opina justamente lo contrario, acusando a Rima de inestable e infiel. Con estos planteamientos les es ciertamente imposible entenderse y comunicarsc, y posiblemente las relaciones entre Marjory y Thaw sufrieran, desde otra óptica, similares problemas.

Lo que es cierto es que ambas relaciones están marcadas por la desconfianza de los personajes femeninos: si bien Marjory no estỉ muy convencida de su interés por Thaw, él la atrae de alguna manera que se la escapa. Rima es mucho más clarn y categórica en su explicación de las dudas de Marjory, y sus propias reservas sobre Lanark. Para Rima, Marjory encontró a Thaw «lunny, embarrasing and not very sexy" y al decir ésta 
alirmación se está identilicando a si misma con ella y a 'Tliaw con Lanark, ya que algo más adelante calilica a Lanark de ordinario y decepcionente, a pesar de haberla salvado de su destrucción en el instituto: "W'here you the only one who could help me, Lanark? Nobody special? Nobody splendid?» (p. 96)

\section{3.-La Expresión Artística Como Forma de Trascendencin:}

En un momento de la narración nuestro «héroe» realiza la caballerosa gesta de salvar a su supuesta dama de las garras de su propia personalidad de dragón. por lo que erit de esperar un cierto reconocimiento a su valor y la debida consideración y gratitud por parte de su pareja. En vez de eso, lo que sucede es que el cuento feliz se viene abajo cuando Lanark no llena los deseos y sueños que centran la búsqueda de Rima. Para colmo de males, el manipulador Sluden reüne a la perfección tales sueños por lo que estä en situación de conseguir a Rima cuando así lo deséce, y elige el momento más doloroso, cuando ella presenta i Lanark el hijo de ambos:

"Lanark said, "Listen Sludden, I want the company of my wife and child do you understand me? »

"Of Course!", said Sludden checrily. «I'm just leaving. I'll come back for you all laters.

"What do you mean?"

"Sludden has offered us a room in his house", said nima.

"We're not taking it». (p. 424)

El despojar a Lanark de su hijo, Alexander o Sandy, ya que hima y él no se ponen de acuerdo ni siquiera en su nombre, es el mayor acto de crueldad que se puede infligir a Lanark, puesto que de la misma manera que Thaw tienc una imperiosa necesidad de crear y expresar su particular visión del mundo a través de sus murales, Lanark necesita desesperadamonte a su hijo para tener algo por lo que luchar, alguien de quien preocuparse y sentirse responsable. En delinitiva, un pedazo de sí mismo que le trascienda y dé un sentido a toda su vida.

$\mathrm{Y}$ es que no hemos de olvidar que, a diferencia de Thaw, Lanark no es un artista, y por tanto es incapaz de orientar sus energías hacia la representación de una creación en la cual el pueda soñar con aparecer como otro dios, con capacidad para hacer y rehacer a su antojo $\left({ }_{6}\right.$ No es exactamente eso lo que está haciendo Gray?):

"Sleeping had become as easy a work, for he dreamed he was in the mural. «lHere it is: land, sky and sunlight, he said to God his lather as they strolled round the bramble bush, the serpent wagging its tail behind them. It was a clear day and anemones were singing in the tidal pools. "You'll get it back when l've put it in decent order. I don't 
like being in debt. As you see l've had no trouble with rational pain and death.m (p. 338)

Esta nueva creación rediseñada desde parámetros eminentemente artísticos no es, sin embargo, suficiente. El «deshiclo" (pues éste es el signllicado de (thaw») que cl arte puede aportar al congelado y paralizante ambiente cultural de Glasgow tiene tan poco impacto sobre la globalidad del sistema como el experimento llevado a cabo por Robert Owen en New Lanark con el objetivo de fundir y neutralizar las fuerzas alienadoras del capitalismo industrial. I'law y Lanark encarnan las posibilidades de transformar la vida cotidiana nediante el arte y la política -en la tradición dialéctica entre lo estélico y lo práctico-sólo para descubrir la realidad de un mundo de pesadilla en cl cual toda ruta de escape conduce inexorablemente hacia otra ramificación monstruosa de un sistema en el cual somos a la vez explotadores y explotados. La gran paradoja en que se sustenta Lanark consiste en que su héroe. Thaw, ha de ser privado del arte como ruta de escape a través de la imaginación,y este proceso se realiza por medio de la vitalidad creativa de la propia obra literaria en la que Gray construye una fantasia que completa la vida de Thaw. Ese nuevo mundo fantástico y surreal es a la vez el lugar de encuentro con la mujer que no pudo obtener en su forma anterior y crítica mordaz a las rutas de escape ante el sufrimiento existencial que ofrece la fantasia sin intentar transformar la realidad circundante, puesto que Thaw renace no como un artista en toda plenitud ropresentando lo más avanzado de la creatividad lumana, sino como un ser normal y corriente, cuya única salida le es de nuevo brindada por la ficción del libro en si mismo.

Si bien Lanark emplea sus fuerzas de manera mis espasmódica que Thaw, una vez que se decide a ponerse en marcha lo hace con convencimiento y determinación, y en este sentido podriamos compararle con una especie de Quijote, empeñado en fantaisticas y absurdas empresas. En otros aspectos nos recuerda al «Everyman», el hombre corriente que se empeña en poner las cosas en orden dentro de si, con Rima, con su hijo y finalmente con su mundo. La razón ültima de Lanark para aceptar la farsa de su misión a Provan reside más en el amor y la preocupación por asegurar un futuro a su hijo que en la seducción de un Sludden a punto de alcunzar la cima de su poder. Y sin embargo, en el mismo momento en que yn no puede hecharse atrás, Lanark reconoce lo absurdo de su sacrificio y el contrasentido que supone su decisión a sabiendas de que le consumirá gran parte de su tiempo vital y prácticamente toda oportunidad de lormar una relación estable con su hijo. Como de costumbre, ya serí demasiado tarde:

"The black shape of someone's head and shoulders looked out of this, and if the window belonged to Sludden's house the watcher was surely Sandy and at once the grotesque flimsy ajreraft and being a delegate and a provost seemed stupid evasions of the realest thing in the world and he shouted «No!m. (p. 467) 
Pero la decisión de Lanark de desplazarse a Provan es de cualquier forma inevitable, ya que Lanark (Como antes que al el padre de Thaw) considera que un hijo justifica y dá cierto sentido a todas las penalidades y sacrilicios, por duros que sean.

Lo que en delinitiva no deja de ser una broma ingeniosa es la libertad con la que Gray nos permite leer y experimentar las historias de Thaw y de Lanark segün varias posibilidades. Podemos suponer que ambas forman substancialmente un único hilo argumental, o bjen podemos considerar la parte realista de Thaw como una versión de hechos reales (autobiografía) o posibles (biografía de ficción) frente a una fábula que refleja nuestros miedos sobre una vida posterior o unos mundos paralelos al nuestro. Igualmente podemos dar por hecho que Thaw efectivamente se alhogó y ése fué su final, o quizá interpretar la escena metalóticamente como una «inmersión» que abre las puertas del mundo del subconsciente o permite traspasar los límites de nuestra roalidad: existen tantas posibilidades como en Pincher Martin de Golding. Quizá la ínterpretación más interesante sea la que considera a los dos protagonistas principales como representaciỏn de la mismi persona en dos estados diferentes, definidos por distintos niveles de consciencia. En este caso, la clave final consiste en que, atunque Thaw y Lanark sean la misma persona en un sentido limitado, debido al paso del tiempo y a los cambios de percepcion que ello produce, ambos son substancialmente personalidades diferentes.

\section{4.-La Redención de Lanark:}

La actitud linal de Lanark, en el contexto general del libro, no se puede reducir sencillamente a un estado de vacío y desilusión. Lanark se ha convertido, linalmente, en persona y mientras que otros personajes como Monboddo se limitan a sometorse y aceptar lo que ocurra, Latnark no se resigna y en una actitud lumana al menos se queja. Thesulta comprensible su petición (lógicamente dirigida al autor) de un mejor linat, algo más especial, lo que no deja de recordarnos las protestas de ltima en otro momento del libro. Sin embargo tampoco en las últimas líncas Gray está dispuesto a hacer concesiones.

Los últimos momentos de Lanark muestran un ligero (y humano) despliege de autocompasión y petulancia, una parodia de la ligura del angel de la muerte que llega para anunciar el final bajo la ligura del chambelán, compuesta del Doctor Munro del instituto y de otro personaje llamado Gloopy. Tal criatura no puede ser una gran ayuda para nadie que esté enlrentándose a sus últimos instantes, si bien es cierto que sí hay algo para lo cual este tipo de apuriciones son tátiles: se les puede liacer permanecer estojcamente escuchando los lamentos finales del moribundo. Latnark no actúa cómicamente en esta ocasión, sino que se inuestra bajo control, y seriamente preocupado por saber quién se hará cargo de su hijo y del mundo cuando él 
no esté. (Quizás un planteamiento sincero pero hasta cierto punto arrogante). Se trata de una pregunta sin respuesta, que el clımbelán lógicamente ignora por lo que Lanark despide amablemente al emisario.

En la mente de Lanark se lia becho la luz ( ¿No éra éste el objetivo de su búsqueda?) hasta el punto de que puede liberarse a sí mismo de sus más oscuros temores, para permanecer receptivo hacia la iuz, ln iluminación personal fruto del targo camino que ha tenido que recorrer. Con un coherente y recientemente adquirido sentido de su dignidad humana, Lanark se dá cuenta de que es hora de irse.

"Lanark forgot him, propped his chin on his laands and sat a long time watching the moving clouds. He was a slightly worried, ordinary oid man but giad to see the light in the sky.m (p. 560)

El «fracaso» linal de Lanark nos conduce de vuelta al comienzo de todo. La derrota que también sufre Thaw en su vida y en su arte, es trascendida por su “renacimiento» como Lanark, puesto que bajo esa nueva forma él puede liegar a comprender su propio mundo a través de las «realidades» fantásticas de otros mundos paralelos al nuestro. El heroismo de Thaw/Lanark está en parte compuesto por una decidida testarudez en la negativa a aceptar que la realidad se rige por designios ajenos $e$ inmutables, una postura mantenida Inciuso en el momento de enfrentarse cara a cara con quien los ha diseñado, un dios-autor que sóio es capaz de ofrecer un linal de destruceión. Lanark. por su parte, ha sido capaz no sólo de alcanzar un elevado grado de humanidad, sino de transmitirlo y perdurar a través de su hijo, quien, ademäs de encarnar un desalín directo al control del autor, se convierte en piedra angular a partir de la cual es posible el encuentro entre Thaw y su padre y con él la plenitud tan arduamente buscada a lo largo de toda la narración.

De esta manera, lo «imaginario» (Lanark) ha redimido a lo «real» (TThaw) y al hacerio ha adquirido una realidad en si mismo que supera a la del autor. Thaw no puede lograr que su arte cobre vida, pero a través de Lanark se le da otra oportunidad para vivir en un entorno en el cual sus mayores pesadilias se han hecho realidad, a pesar de lo cual existe la posibilidad de lograr una plenitud sexual que siempre ie fue negada en su vida anterior y que lleva implícita la creación de vida. El momento culmen de la redención de Thaw se produce en una escena que conjuga y funde las perspectivas de una dobie visión, mezclando recuerdos de Thaw en relación a su padre con los de Alexander por parte de Lanark:

«Lanark sighed with pleasure, halted and looked away in the biue. He said. "Thank you!» and lor a moment giimpsed the gost of a man scribbling in a bed littered with papers. Lanark smiled and said, «No, oid Nastier, it isn't you I thank, but the cause of the ground which grew us alt. I have never given you much thougith, $\mathrm{Mr}$. cause...and on the whole I have found your world bearable rather 
than good. (...) I don't care when contentment ends. I don ${ }^{\dagger} t$ care what absurdity, failure, deatlı l am moving toward. Even when your world has lapsed into black nothing, it will have made sense because Sandy once enjoyed it in the sunlight. I am not speaking for mankind. If the poorest orphan in creation has reason to curse you, then everything high and decent in you should go to Hell. Yes! Go to Hell, go to Hell, go to I.Jell as often as there are victims in your universe. But I am not a victim. This is my best moment.» (p.515)

Precisamente a la luz de esta revelación, los marcados paralelismos y las frecuentes repeticiones en las que Thaw y Lanark se encuentran atrapados a lo largo de la narración adquieren un ma tiz redentor. La figura que Lanark contempla subiendo la colina es a la vez cl joven Thaw y su propio hijo Sandy quicn, en un juego de palabras, proporciona "sandy tocholes" para que Lanark recupere su infancia como Thaw y éste a su vez alcance, a través de Lanark, la capacidad de procreación que le fue negada en su vida. Thaw y Lanark, padre e hijo, realismo e imaginación. historia y atemporalidad se funden en este instante y el espectador mira a la vez al valle desde la cima y a la cima desde el valle. A pesar de las contínuas derrotas, de las caídas progresivas hacia mundos cada vez más infernales, Lanark alcanza un cierto nivel de éxito y se convierte en uno de nosotros, entre el sueño y la pesadilla, sobreviviendo a la tirania que impone la realidad, y esperando tranquilamente el linal.

Es posible, por tanto, encontrar un lugar para la esperanza en el final de Lanark, puesto que la postura última del protagonista ante su destino se halla muy próxima a lo que Alasdair Gray denomina "stoical loneliness", un estado de soledad que se constituye en puerta abierta para explorar el entendimiento entre los seres humanos:

«My characters are stoics resigned to lonelyness because I think everyone is essentially that. Stoical lonelyness is only a tragic state if it has turned callous or hopeless. If it has not, then it is the best foundation for co-operation, and even love, between people of different sexes, classes or culturess't

Lanark es, en delinitiva, un brillante ejercicio de ficción imaginativa, a través del cual somos capaces de reflexionar sobre los términos de nuestra existencia, mediante el análisis de nuestro acamino" a través de una realidad en contínuo cambio. La novela lleva il cabo magistralmente el proceso de transformación de lo real gracius al personaje de Lanark/Thaw, permitiendo a Gray construir, al final de la obra, una alegoria política que resume en los mecanismos de tan grotescos mundos Ia trama de nuestra propia roalidad.

${ }^{1+}$ Anderson, Carol; Cencrastus Revitw, N"13, p.6 